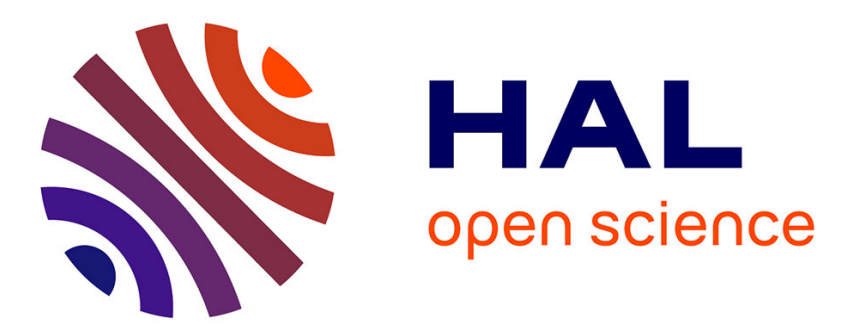

\title{
A rapid and sensitive method for characterization and quantification of polyglycerol esters by supercritical fluid chromatography coupled to high-resolution mass spectrometry (SFC-HRMS).
}

Thi Phuong Thuy Hoang, Nicolas Ritter, Jean-David Rodier, David Touboul

\section{- To cite this version:}

Thi Phuong Thuy Hoang, Nicolas Ritter, Jean-David Rodier, David Touboul. A rapid and sensitive method for characterization and quantification of polyglycerol esters by supercritical fluid chromatography coupled to high-resolution mass spectrometry (SFC-HRMS).. Talanta, 2021, 230, pp.122316. 10.1016/j.talanta.2021.122316 . hal-03206013

\section{HAL Id: hal-03206013 \\ https://hal.science/hal-03206013}

Submitted on 14 Oct 2021

HAL is a multi-disciplinary open access archive for the deposit and dissemination of scientific research documents, whether they are published or not. The documents may come from teaching and research institutions in France or abroad, or from public or private research centers.
L'archive ouverte pluridisciplinaire HAL, est destinée au dépôt et à la diffusion de documents scientifiques de niveau recherche, publiés ou non, émanant des établissements d'enseignement et de recherche français ou étrangers, des laboratoires publics ou privés. 


\section{A rapid and sensitive method for characterization and quantification of polyglycerol esters by Supercritical Fluid Chromatography coupled to High-Resolution Mass Spectrometry (SFC-HRMS)}

Thi Phuong Thuy Hoang ${ }^{1}$, Nicolas Ritter ${ }^{2}$, Jean-David Rodier ${ }^{2}$, David Touboul ${ }^{1 *}$

${ }^{1}$ Université Paris-Saclay, CNRS, Institut de Chimie des Substances Naturelles, UPR 2301, 91198, Gif-sur-Yvette, France

${ }^{2}$ Gattefossé, 36 chemin de Genas, CS70070, 69804 Saint Priest cedex, France

*Corresponding author:

David Touboul, Université Paris-Saclay, CNRS, Institut de Chimie des Substances Naturelles, UPR 2301, 91198, Gif-sur-Yvette, France.

Phone number: +33-1-69-82-30-32

Email: david.touboul@cnrs.fr 


\begin{abstract}
Polyglycerol esters of fatty acids (PGEs), a very complex mixture of various isomers, are widely used as green surfactants in different industrial fields such as in cosmetic, pharmaceutic and food industries. However, no study related to the purification and the absolute quantification of these compounds has been described yet. In this study, we developed a rapid and efficient method for characterization and quantification of PGEs using Supercritical Fluid $\mathrm{CO}_{2}$ coupled to High-Resolution Mass Spectrometry (SFC-HRMS). The SFC conditions were first considered including the stationary phase, the nature of mobile phase, the column temperature, the back-pressure regulator. The MS parameters (drying-gas temperature, capillary voltage, nozzle voltage, fragmentor voltage) were then investigated to get the best sensitivity for the PGE analysis. The MS/MS-based structural characterization of targeted PGE, triglycerol mono-oleate (PG3+1C18:1), was established and is helpful to study complex mixtures of PGEs with numerous isobaric PGEs. PG3+1C18:1 was then purified at lab scale and used as standard for quantification. This enabled to develop a rapid quantification method for PG3+1C18:1 within 12 min with good linearity $\left(R^{2}=0.9997\right)$ as well as sensitivity (picogram level). The validated method was then successfully applied to quantify PG3+1C18:1 in commercial products in order to evaluate their composition.
\end{abstract}

\title{
Keywords
}

polyglycerol esters; surfactant; supercritical fluid chromatography; high resolution tandem mass spectrometry; quantification 


\section{Introduction}

Polyglycerol esters (PGEs, Fig. 1) are a class of non-ionic emulsifiers which are extensively used in different industries including cosmetics, pharmaceuticals and food industries. Their interesting features such as broad range of polarity or hydrophilic-lipophilic balance number (HLB) values make them a highly valuable emulsifier [1]. Further promising physical-chemical properties are also found in this class of compounds as odorless, biodegradable, good thermal stability and moderate antimicrobial activity. These properties lead to widespread applications of PGEs in the industry [1-3].

PGEs are obtained by the synthesis from glycerol and fatty acid through 2 steps. The first one is the polymerization of glycerol, which generally come from the biodiesel process as a by-product [4] or fatty acid production, resulting in a complex mixture of polyglycerol oligomers $[1,5]$. These oligomers could be linear, branched or cyclic. This mixture is then esterified in the second step with one or several fatty acids giving PGEs. When polyglycerol oligomers are available, PGEs could be directly formed by mixing them with fatty acids. The final product is very complex with various compounds different in the degree of polymerization, the branching type of oligomers, the number and the length of fatty acids [6]. Therefore, a method for the PGE qualification and/or quantification would be necessary to optimize the synthesis process. Furthermore, as the composition of a mixture designs its properties, it is worth to characterize as well as quantify the PGE mixture.

However, the similar physical-chemical properties of PGEs as well as their non-UV-absorbing behavior make difficult to purify and analyze these compounds. Furthermore, some PGEs are exhibiting chiral carbons leading to numerous stereoisomers in mixture [7]. Thus, no absolute quantitative method for PGEs has been described yet in the literature. Some studies about PGE analysis methods have been published by using thin-layer chromatography (TLC) [8], gas chromatography (GC) [9], high-performance liquid chromatography HPLC [6, 7, 10], GC combined to LC [11], supercritical fluid chromatography (SFC) [12] with mass spectrometry (MS) [6, 7, 9-11], evaporative light scattering detection (ELSD) [7], or flame ionization detector (FID) [12]. Recently, a (U)-HPLC-MS method for relative PGE quantification has been developed but without taking into account the difference in ionization yields of PGEs depending on their structures [6].

In recent years, SFC in analytical chemistry has attracted much attention thanks to its numerous advantages such as short run time analysis, high efficiency and low organic solvent consumption [13-15]. The addition of a polar solvent in the mobile phase with/without additive at low concentration allows analyzing compounds with wide polarity range. Furthermore, SFC has been shown to be performant in chiral separation $[16,17]$. Thus, it would be interesting to use SFC to separate PGE mixtures.

Herein, we present a method using SFC coupled to high-resolution mass spectrometry (SFC-HRMS) to analyze PGEs and to establish the MS/MS-based structural characterization of this class of compounds. After a column screening performed on nine different stationary phases, the SFC conditions (nature of cosolvent, column temperature, back-pressure regulator (BPR) and MS parameters (drying gas temperature, capillary voltage, nebulizer voltage, fragmentor voltage) were optimized. A PGE purification method using HPLC was also introduced allowing to purify triglycerol mono-oleate PG3+1C18:1. This enabled for the first time to develop an absolute quantitative method for this particular ester by SFC-HRMS. 


\section{Materials and methods}

\section{General experimental procedures}

1D and 2D NMR spectra were recorded in $\mathrm{MeOH}-\mathrm{D}_{4}$ on Bruker Avance $500 \mathrm{MHz}$ spectrometers. Chemical shifts were referenced using residual solvent $\left(\delta_{\mathrm{H}} 3.31 \mathrm{ppm}\right.$ and $\left.\delta_{\mathrm{C}} 49.15 \mathrm{ppm}\right)$.

\section{Synthesis}

The following procedure was applied to synthetize polyglyceryl-3 esters PG3MO: Starting materials including $3.15 \mathrm{~g}$ of oleic acid (99\%, Larodan, USA), $5.12 \mathrm{~g}$ of commercial polyglycerol-3 (Spiga spa, Italy) and $0.08 \mathrm{~g}$ of sodium hydroxide (30\%, Alfa Aesar, Germany) were charged into a $25 \mathrm{~mL}$ pyrex tube equipped with a PTFE pipe designed for water elimination. The resulting mixture was stirred at $220{ }^{\circ} \mathrm{C}$ under a nitrogen flow $\left(1 \mathrm{~L} \mathrm{~min}^{-1}\right)$. After $7 \mathrm{~h}$ of stirring, the mixture was decanted at $80{ }^{\circ} \mathrm{C}$ for $3 \mathrm{~h}$ affording PG3MO.

Samples PG3DO1 and PG3DO2 are two different batches of commercial polyglyceryl-3 dioleate supplied by Gattefossé (Plurol® oleique CC 497).

\section{Chemicals}

Carbon dioxide $\left(\mathrm{CO}_{2}\right.$, purity $\left.\geq 99.7 \%\right)$ was purchased from Air Liquide (Grigny, France). Methanol (MeOH, LC-MS grade) and acetonitrile (ACN, ultra-gradient HPLC grade) were purchased from J.T. Baker (Center Valley, PA, USA). ACN (HPLC grade), dichloromethane $\left(\mathrm{CH}_{2} \mathrm{Cl}_{2}\right.$, stabilized with amylene) and ethanol absolute anhydrous (EtOH, HPLC-Isocratic grade) were purchased from Carlo Erba (Val de Reuil, France). Ammonium acetate (AcONH 4 , LC-MS grade) was purchased from Sigma-Aldrich (Saint-Quentin Fallavier, France). Water was purified on a Millipore direct-Q apparatus (MilliporeMerck, Alsace, France). Three products were provided by the Gattefossé company: PG3MO, PG3DO1 and PG3DO2. Product PG3DO2 was used to study the optimal SFC-HRMS and SFC-HRMS/MS conditions at a concentration of $1 \mathrm{mg} \mathrm{mL} \mathrm{m}^{-1}$ in $\mathrm{MeOH} / \mathrm{CH}_{2} \mathrm{Cl}_{2}$ 1:1 v/v. Product PG3MO was used for the purification of PG3+1C18:1. Solutions of $\mathrm{PG} 3+1 \mathrm{C} 18: 1$ used in method validation section were prepared from a stock solution $(0.1 \mu \mathrm{g}$ $\mathrm{mL}^{-1}$ in $\mathrm{MeOH}$ ) and stored at $-20{ }^{\circ} \mathrm{C}$.

\section{SFC}

Analyses were performed on a 1260 Infinity Analytical SFC system (Agilent Technologies, Waldbronn, Germany) consisting of an Aurora module and an "LC-like" system. This system was equipped with a thermostated autosampler (kept at $5{ }^{\circ} \mathrm{C}$ ) with a $5 \mu \mathrm{L}$ injection (full loop mode with overfill factor of 3 ) and two thermostated column compartments that can contain up to eight columns.

Nine columns were selected for the screening of the stationary phase. Three columns were purchased from Waters (Guyancourt, France): Torus Acquity UPC ${ }^{2} 1$-aminoanthracene (1-AA) $(50 \mathrm{~mm} \times 2.1 \mathrm{~mm}, 1.7 \mu \mathrm{m})$, TorusAcquity UPC ${ }^{2}$ diethylamine-bonded silica (DEA) $(50 \mathrm{~mm} \times 2.1 \mathrm{~mm}, 1.7 \mu \mathrm{m})$ and Acquity $\mathrm{UPC}^{2} \mathrm{BEH}$ 2-ethylpyridine (2-EP) $(100 \mathrm{~mm} \times 2.1 \mathrm{~mm}, 1.7 \mu \mathrm{m})$. The Hypercarb ${ }^{\mathrm{TM}}(100 \mathrm{~mm} \times 2.1 \mathrm{~mm}, 5 \mu \mathrm{m})$ was purchased from Thermo Fisher Scientific (Courtaboeuf, France). Four columns were purchased from Agilent Technologies (Massy, France): ZORBAX Eclipse Plus C18 - Rapid Resolution HT (C18) (150 mm $\times 2.1 \mathrm{~mm}, 1.8 \mu \mathrm{m})$, Pursuit 3 pentafluorophenyl (PFP) $(150 \mathrm{~mm} \times 2 \mathrm{~mm}, 3 \mu \mathrm{m})$, Pursuit XRs Diphenyl (DP) $(250 \times 2 \mathrm{~mm}, 3 \mu \mathrm{m})$ and Zorbax RRHD SB-CN $(\mathrm{CN})(100 \mathrm{~mm} \times 2.1 \mathrm{~mm}, 1.8 \mu \mathrm{m})$. The Amine Luna $\mathrm{NH}_{2}\left(\mathrm{NH}_{2}\right)(150 \mathrm{~mm} \times 2 \mathrm{~mm}, 3 \mu \mathrm{m})$ was purchased from Phenomenex (Le Pecq, France). The DEA column was selected for the quantification step.

The mobile phase was composed of $\mathrm{CO}_{2}$ (A) and co-solvent (B). For the screening of stationary phase, the mixture of $\mathrm{MeOH} / \mathrm{EtOH} \mathrm{1:1} \mathrm{v/v} \mathrm{with} 20 \mathrm{mM}$ of $\mathrm{AcONH}_{4}$ was employed as co-solvent. The flow rate was fixed at $1 \mathrm{~mL} \mathrm{~min}{ }^{-1}$. Run time of 16 min was programed as follows: 0-10 $\min$ (1-30\% B), 10-12 $\min (30 \%$ 
B), 12-13 $\min \left(30-1 \%\right.$ B), 13-16 $\min (1 \% \mathrm{~B})$. The column temperature was $50{ }^{\circ} \mathrm{C}$. The BPR was set at 130 bar and $60{ }^{\circ} \mathrm{C}$. The make-up solvent consisted of MeOH/EtOH 1:1 v/v with $20 \mathrm{mM}$ of $\mathrm{AcONH}_{4}$ and its flow rate was set at $0.2 \mathrm{~mL} \mathrm{~min}^{-1}$.

To get the optimum SFC separation, the gradient, the nature of co-solvent together with different values of column oven temperature $\left(40,50\right.$ and $\left.60^{\circ} \mathrm{C}\right)$ and $\mathrm{BPR}$ pressure $(130,140$ and $150 \mathrm{bar}$ ) were examined.

\section{SFC-HRMS analysis}

The SFC system was coupled to a quadrupole time-of-flight (QTOF) mass spectrometer 6540 Agilent (Agilent Technologies, Massy, France) equipped with an electrospray ionization ESI dual JetStream. After the UV detector, a make-up solvent was added into the mobile phase to improve the solubility of analytes. This solvent was pumped by a 1260 Infinity isocratic pump (Agilent Technologies, Massy, France) with a flow rate of $0.2 \mathrm{~mL} \mathrm{~min}^{-1}$. Before the ion source entrance, a Caloratherm (Sandra Selerity Technologies, Kortrijk, Belgium) fixed at $60{ }^{\circ} \mathrm{C}$ was used to avoid the freezing of the transfer line.

MS parameters were set as follows: drying gas at $10 \mathrm{~L} \mathrm{~min}^{-1}$, nebulizer at $50 \mathrm{psi}$, sheath gas temperature at $350{ }^{\circ} \mathrm{C}$, sheath gas flow at $11 \mathrm{~L} \mathrm{~min}^{-1}$, nozzle voltage at $1000 \mathrm{~V}$, skimmer at $45 \mathrm{~V}$, Oct $1 \mathrm{RF} \mathrm{Vpp}$ at $750 \mathrm{~V}$. Other parameters including fragmentor voltage, drying-gas temperature, capillary voltage (Vcap) and nozzle voltage were further investigated to get the optimal values. Experiments were carried out in positive ionization mode. Full-scan mode from $m / z 50$ to $\mathrm{m} / z, 1700$ at $2 \mathrm{GHz}$ was selected resulting in a mass resolution of 26000 at $\mathrm{m} / \mathrm{z}$ 922. Calibration solution containing two internal reference masses (purine, $\mathrm{C}_{5} \mathrm{H}_{4} \mathrm{~N}_{4}, \quad m / z \quad 121.0509$, and HP-921 [hexakis-( $1 \mathrm{H}, 1 \mathrm{H}, 3 \mathrm{H}$-tetrafluoro pentoxy)phosphazene], $\mathrm{C}_{18} \mathrm{H}_{18} \mathrm{O}_{6} \mathrm{~N}_{3} \mathrm{P}_{3} \mathrm{~F}_{24}, \mathrm{~m} / z$ 922.0098) purchased from Agilent (Agilent Technologies, Massy, France) was injected routinely in order to get mass accuracy below 5 ppm. MassHunter Workstation software (B.08.00) was used to analyze the recorded data. For each ion of interest, the extracted ion chromatogram (EIC) was traced by selecting the theoretical $\mathrm{m} / \mathrm{z}$ value with a selection window of $20 \mathrm{ppm}$.

\section{SFC-HRMS quantification}

Optimal SFC and MS parameters were set for the PG3+1C18:1 quantification method. Run time was reduced to 12 min including column stability time. The DEA column temperature was $50{ }^{\circ} \mathrm{C}$. The injection volume was fixed at $5 \mu \mathrm{L}$. The mobile phase was composed of $\mathrm{CO}_{2}(\mathrm{~A})$ and $\mathrm{MeOH}(\mathrm{B})$ with a flow rate of $1.2 \mathrm{~mL} \mathrm{m^{-1 }}$. The gradient was: 0-7 $\min (6-15 \% \mathrm{~B}), 7-9 \min (15 \% \mathrm{~B}), 9-9.1 \mathrm{~min}(15-6 \% \mathrm{~B}), 9.1-12 \mathrm{~min}$ (6\% B). The BPR was set at 140 bar and $60{ }^{\circ} \mathrm{C}$. The make-up solvent was $\mathrm{MeOH}$ with a flow rate of 0.2

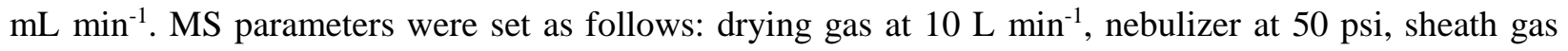
temperature at $350{ }^{\circ} \mathrm{C}$, sheath gas flow at $11 \mathrm{~L} \mathrm{~min}^{-1}$, Vcap $3500 \mathrm{~V}$, nozzle voltage at $1000 \mathrm{~V}$, fragmentor $200 \mathrm{~V}$, skimmer at $45 \mathrm{~V}$, Oct $1 \mathrm{RF}$ Vpp at $750 \mathrm{~V}$.

\section{SFC-HRMS/MS analysis}

SFC conditions and MS scans were set as indicated above. The fragmentor voltage and the drying-gas temperature were studied to optimize the MS/MS analyses. The mass range of precursor and fragment ion was set between $\mathrm{m} / \mathrm{z}, 100$ and $\mathrm{m} / \mathrm{z}$ 1200. Selected parent ions ( $\mathrm{m} / \mathrm{z} 505.3735,579.4103,653.4471)$ were fragmented at $10 \mathrm{eV}$ of collision energy with a mass isolation window of $1.3 \mathrm{amu}$.

\section{SFC purification of PG3+1C18:1}

Semi-preparative SFC was carried out on an Investigator SFC System (Waters) equipped with a diode array detector (DAD) and an ELSD. The diphenyl column $(250 \mathrm{~mm} \times 4.6 \mathrm{~mm}, 5 \mu \mathrm{m})$ purchased from Agilent Technologies (Massy, France) was employed. The DAD was set at 210 and $254 \mathrm{~nm}$. Product PG3MO (100 $\mathrm{mg} \mathrm{mL}{ }^{-1}$ in $\mathrm{MeOH} / \mathrm{CH}_{2} \mathrm{Cl}_{2} 1: 1 \mathrm{v} / \mathrm{v}$ ) was subjected to $\mathrm{SFC}$ with an injection volume of $60 \mu \mathrm{L}$. The column temperature was set at $40{ }^{\circ} \mathrm{C}$. The $\mathrm{BPR}$ was at 130 bar. The mobile phase consisted of $\mathrm{CO}_{2}$ (A) and $\mathrm{MeOH} / \mathrm{EtOH} \mathrm{1:1} \mathrm{v/v} \mathrm{(B)} \mathrm{with} \mathrm{a} \mathrm{flow} \mathrm{rate} \mathrm{of} 4 \mathrm{~mL} \mathrm{~min}^{-1}$. The gradient was: 0-15 $\min (8 \% \mathrm{~B}), 15-18 \mathrm{~min}$ 
(8-30\% B), 18-23 $\min (30-30 \%$ B), 23-25 $\min$ (30-8\% B), 25-29 min (8\% B). The make-up solvent was $\mathrm{MeOH} / \mathrm{EtOH}$ 1:1 v/v with a flow rate of $3 \mathrm{~mL} \mathrm{~min}^{-1}$. Six fractions (SFC-F1 to 6) were collected and dried under nitrogen at ambient temperature.

\section{HPLC purification of PG3+1C18:1}

HPLC purification was performed on a Gilson system equipped with a 322 pumping device, a GX-271 fraction collector, a $171 \mathrm{DAD}$ and a prepELSII electrospray nebulizer detector. The DAD was set at 210, 220, 254, 272 and $310 \mathrm{~nm}$. A Luna $\mathrm{C}_{18}$ column ( $250 \mathrm{~mm}$ x $21.1 \mathrm{~mm}, 5 \mu \mathrm{m}$, Phenomenex) was employed. The mobile phase consisted of $\mathrm{H}_{2} \mathrm{O}(\mathrm{A})$ and $\mathrm{ACN}(\mathrm{B})$ with a flow rate of $21 \mathrm{~mL} \mathrm{~min}^{-1}$. The gradient was: 0-15 $\min (85 \%$ B), 15-15.5 $\min (85-100 \%$ B), 15.5-23 $\min (100 \%$ B), 23-24 $\min (100-85 \%$ B), 24-30 min (85\% B). Product PG3MO (50 mg mL $\mathrm{mL}^{-1}$ in $\left.\mathrm{ACN} / \mathrm{H}_{2} \mathrm{O} 1: 1 \mathrm{v} / \mathrm{v}\right)$ was subjected to HPLC purification with an injection volume of $400 \mu \mathrm{L}$ affording pure PG3+1C18:1 (5.8 mg).

\section{Method validation}

The selectivity of the method was studied by adding the mixture of polyglycerol and oleic acid used for the synthesis to the standard solution.

Linear least squares regression was calculated based on the peak area of the analyte at six different levels (0.5-20.0 $\left.\mathrm{ng} \mathrm{mL}^{-1}\right)$ in triplicate experiments. The slope, the $y$-intercept of the calibration curve as well as the coefficient of determination $\left(R^{2}\right)$ were calculated.

The limits of detection (LOD) and quantification (LOQ) were defined as the concentration with a signalto-noise ratio $(\mathrm{S} / \mathrm{N})$ of 3 and 10 , respectively [18].

To study intra- and inter-day precision, six calibration levels were injected in triplicate in a single run and in three different days. The relative standard deviation (RSD), the back-calculated concentration as well as the accuracy were determined.

Application of the validated method

The quantification of PG3+1C18:1 in three products was performed using the validated method, based on the established external calibration curve.

\section{Results and discussion}

\section{Optimization of SFC separation}

First, SFC parameters including the chemistry of the stationary phases, the mobile phase composition and the column temperature were studied in order to separate and detect a large variety of PGEs. Table S1 in supplementary data displays 50 PGEs potentially expected in the commercial mixture. These molecules could be mono-, di-, tri-, tetra-, penta-esters of oleic acid and polyglycerols with a polymerization degree ranging from 2 to 6 , and their analogs with one loss of water molecule. The esters of glycerol and polyglycerol having degrees of polymerization higher than 6 were not detected. The mass range acquisition was set at $m / z$ 100-1700 so the detection was limited up to penta-esters of polyglyceryl-3 esters. Targeted compound in this study is PG3+1C18:1, which is major in the commercial product.

To study the influence of the stationary phases on the separation, the PG3DO2 solution was analyzed on nine different columns using a generic gradient (Fig. 2). When using the Hypercarb column, no PGE was detected. Surprisingly, a previous study had showed that the Hypercarb column allowed to efficiently separate PGEs diastereoisomer by HPLC [7]. This difference could be explained by the difference in the mobile phase condition, liquid versus supercritical, which could influence the affinity between the analyte and the stationary phase. The PGEs were mainly co-eluted on the PFP, CN and C18 columns. They were better separated on the other columns, i.e. 2-EP, 1-AA, DP, $\mathrm{NH}_{2}$ and DEA. 
Among 50 hypothetic PGEs in Table S1, 40 PGEs were detected (PGEs annotated in black bold, Table S1, supplementary data). The DEA enabled the best separation of all PGEs within 8 min so it was selected for the following optimization steps. The higher the degree of polymerization of polyglycerol, the more molecules were retained on column (Fig. 3-a). On the contrary, molecules bearing more esterified oleic acid were eluted earlier (Fig. 3-b). The PGEs having one water loss were eluted before their analogs without water loss (Fig. 3-c) suggesting that the loss of one water molecule was a product of an intramolecular condensation induced during the synthesis procedure. This could be an intrinsic cyclic or a dehydration phenomenon.

Furthermore, the DP column enabled to separate PGEs in class while the DEA separated them in class and in species (Fig. S1, supplementary data). It would thus be interesting to use these two columns to isolate PGEs in a successive procedure: the first step to separate PGEs in class by using the DP column, the fractions of interest would be further purified on the DEA column to access pure species.

The mobile phase composition as well as its flow rate were then studied. As mentioned above, the molecules of interest in this study were isomers of PG3+1C18:1. Thus all improvement steps to reach the best separation and detection were focused on these molecules. When using the generic SFC method, different isomers of PG3+1C18:1 were co-eluted (Fig. S2, supplementary data). Furthermore, we observed that all PGEs in the PG3DO2 were eluted between 4 and $15 \%$ of co-solvent. The gradient was then modified by decreasing the gradient slope through a gradient of $6-15 \%$ of co-solvent during $15 \mathrm{~min}$. This led to a better separation of the 3 detected PG3+1C18:1 isomers. The flow rate of the mobile phase was slightly increased from 1 to $1.2 \mathrm{~mL} \mathrm{~min}^{-1}$ to decrease retention times and improve peak shapes. The nature of co-solvent was also examined. As the presence of $\mathrm{AcONH}_{4}$ in co-solvent was not favorable for the purification procedure, a study on the nature of co-solvent was proceeded by using a mixture of $\mathrm{MeOH} / \mathrm{EtOH} \mathrm{1:1} \mathrm{v/v,} \mathrm{pure} \mathrm{MeOH}$ or pure EtOH. As expected, $\mathrm{MeOH}$ enabled the PGEs to elute earlier than the mixture of $\mathrm{MeOH} / \mathrm{EtOH} 1: 1$ $\mathrm{v} / \mathrm{v}$ and pure $\mathrm{EtOH}$. We observed that the chromatographic peaks and the sensitivity were improved when using $\mathrm{MeOH}$. Data (Fig. S2, supplementary data) also showed that $\mathrm{AcONH}_{4}$ did not demonstrate significant improvement of separation and detection of PGEs by SFC-HRMS. MeOH was thus selected as co-solvent.

It has been demonstrated that the column temperature affects the state and the density of the mobile phase, resulting in significant influence on the SFC analyses including changes in retention time, peak shape and method sensitivity [19]. Optimization of the oven temperature was then studied at three different temperatures, i.e. 40,50 and $60{ }^{\circ} \mathrm{C}$. The results showed that setting oven temperature at 50 and $60{ }^{\circ} \mathrm{C}$ enabled a better sensitivity for PG3+1C18:1 (Fig. 4-a). This phenomenon has been observed in a previous study [19]. It should be related to the supercritical state of the mobile phase at high temperature, which resulted in better detection of the analytes by ESI-HRMS. The column temperature was thus kept at $50{ }^{\circ} \mathrm{C}$ to avoid potential column or compound degradation. The BPR was optimized with three pressure levels including 130, 140 and 150 bar. BPR at 140 bar offered a slight improvement in sensitivity of PG3+1C18:1 (Fig. 43-b) and was consequently chosen for further developments.

\section{Optimization of MS parameters}

Different MS parameter settings were optimized to get the best sensitivity for the PGE analysis. First, the drying-gas temperature was studied at three temperatures $\left(250,300\right.$ and $\left.350{ }^{\circ} \mathrm{C}\right)$. The results presented in Fig. 5 show that the PGE peaks got the highest intensity at $350{ }^{\circ} \mathrm{C}$. As a result, this value was kept as optimum. Other compound-dependent parameters were also examined including the Vcap, the fragmentor voltage, the nozzle voltage. The results showed that the Vcap did not present significant influence on the PGE analysis. A value of $3500 \mathrm{~V}$ was selected for this parameter. For the fragmentor voltage, the more important this value, the higher intensity of the PGEs. This parameter was thus kept at $200 \mathrm{~V}$. In contrast 
to the previous parameters, the nozzle voltage did not show a linear influence on the intensity of the PGE signals. At the highest tested value, i.e. $2000 \mathrm{~V}$, intensities of PGE signals were smaller compared to intensities at $1000 \mathrm{~V}$. That could be due to an in-source fragmentation of PGEs at high nozzle voltage. Nozzle voltage at $1000 \mathrm{~V}$ enabled the best sensitivity of the PGEs so it was kept as optimum.

\section{SFC-HRMS/MS characterization of PGEs}

The SFC-HRMS/MS-based characterization of PGEs, especially PG3+1C18:1, was then explored. The PGEs had three molecular ion forms: the minor form is the protonated molecule, the second one is the ammonium cationized species and the major one is the sodium cationized species. Since the protonated species is fragmented at the lowest energy and contains more characteristic fragments than the two others, it was used for the structural characterization study.

First, the SFC-HRMS/MS method was set using the optimum parameters described previously with the collision energy at $0 \mathrm{eV}$. We observed that all three isomers of PG3+1C18:1 were fragmented (Fig. S3, supplementary data) due to residual internal energies of the precursor ions. Our goal was then to minimize this activation process by reducing the fragmentor voltage. The results showed that the fragmentor voltage at 80,100 and $120 \mathrm{~V}$ induced the same proportion of PG3+1C18:1 fragmentation even if the in-source fragmentation was less pronounced when decreasing this parameter value (Fig. S4, supplementary data). Therefore the fragmentor voltage was set at $120 \mathrm{~V}$ for HRMS/MS characterization study..

The collision energy was then increased to $10 \mathrm{eV}$ leading to extended fragmentation of each isomer with similar fingerprints (Fig. 6 and S5). However, their relative intensity was different from one compound to the other. Losses of one, two and three glycerol moieties were observed. The fragments corresponding to free mono-, di-, tri-glycerol together with oleic acid were also present. The consecutive losses of water molecule were detected for each ion. When using our SFC-HRMS/MS conditions to analyze other PGEs in the mixture, such as PG4+1C18:1 and PG5+1C18:1, the same fragmentation pathway was observed (Fig. S6 and S7, supplementary data) indicating the efficiency of the method for PGE structural characterization. As the PGE mixtures are complex and sometimes they could contain many isobaric molecules, such as $\mathrm{PG} 3+\mathrm{C} 18+\mathrm{C} 18: 2$ and PG3+2C18:1, the determination of each PGE type presents a challenge. By using our method, the MS/MS-based fragmentation will allow to characterize each PGE type and distinguish the isobaric PGEs.

\section{Purification and structure elucidation of PG3+1C18:1}

As previously indicated, the two columns DEA and DP showed interest in PG3+1C18:1 purification by SFC. Product PG3MO rich in PG3+1C18:1 was first fractionated by SFC on the DP column affording 6 fractions. SFC-HRMS analyses of these fractions showed an abundant intensity of free triglycerol (PG3), tetraglycerol (PG4) and dehydrated tetraglycerol (PG4(1)) in all fractions. Fraction SFC-2 was almost composed of these molecules and a very low quantity of PG3+1C18:1 (Fig. S8, supplementary data). By comparing SFC-HRMS profiles of PG3MO and corresponding SFC purified fraction, an important decrease of PG3+1C18:1 peak intensity was observed whereas the quantity of free polyglycerols was significantly increased. We supposed that a hydrolysis of PGEs occurred during $\mathrm{CO}_{2}$ decompression and methanolysis/ethanolysis reaction with residual solvents [20].

The purification of PG3+1C18:1 from PG3MO on reversed-phase HPLC was then investigated. After one step of purification, a fraction containing PG3+1C18:1 (5.8 mg) was isolated as a colorless emulsion. The purity of this fraction was first verified by SFC-HRMS (Fig. S9, supplementary data). The MS spectrum of the peak eluted between 3-4 min displayed a major ion peak at $m / z$ 527.3543, for which the molecular formula $\mathrm{C}_{27} \mathrm{H}_{52} \mathrm{O}_{8}$ was deduced $\left([\mathrm{M}+\mathrm{Na}]^{+}, \Delta+3.22 \mathrm{ppm}\right)$. According to Table $\mathrm{S} 1$ (supplementary data), this ion can only be attributed to PG3+1C18:1. A peak shoulder was detected suggesting that this fraction 
contained two PG3+1C18:1 isomers named 1 (major) and 2 (minor). The percentage of isomer 2 calculated based on the peak area was estimated to $13 \%$. In order to confirm the identification of PG3+1C18:1, 1D and 2D NMR experiments were performed (Fig. S10 to S15, supplementary data). Oleic acid and mixture of polyglycerol used for the synthesis were also analyzed by ${ }^{1} \mathrm{H}$ and ${ }^{13} \mathrm{C}$ NMR in the same condition. The comparison of ${ }^{1} \mathrm{H}$ and ${ }^{13} \mathrm{C}$ NMR spectra of these compounds allowed to identify proton and carbon signals belonging to the triglycerol head and to the fatty chain moiety. On ${ }^{1} \mathrm{H}$ NMR spectrum (Fig. S10, supplementary data), the total of proton integration at $\delta_{\mathrm{H}} 2.35 \mathrm{ppm}(1.61)$ and $\delta_{\mathrm{H}} 2.17 \mathrm{ppm}(0.43)$ was of 2.04 suggesting that these protons corresponded to two isomers of PG3+1C18:1 as detected by SFC-HRMS. Because of the weak 2D NMR intensity of minor isomer 2, only the structure of major isomer 1 of PG3+1C18:1 was established. All observed HSQC, HMBC, COSY and NOESY correlations confirmed the distribution of proton and carbon. HMBC spectrum showed the correlation between carbon of a carbonyl group $\left(\delta_{\mathrm{C}} 175.6 \mathrm{ppm}\right)$ and 2 protons of a methylene group $\left(\delta_{\mathrm{H}} 4.06-4.17 \mathrm{ppm}, \delta_{\mathrm{C}} 66.7 \mathrm{ppm}\right)$ suggesting that the ester function was at position 1'. This result is in good agreement with the hypothesis that the esterification was preferred at the terminal hydroxyl group of PG3 [21].

\section{Quantification}

The quantification of PG3+1C18:1 was performed on the basis of the peak area of the pseudo-molecular ion $[\mathrm{M}+\mathrm{Na}]^{+}$. When comparing the peak area of $\mathrm{PG} 3+1 \mathrm{C} 18: 1$ in the standard solution and in the solution added with polyglycerol and oleic acid, no significant difference was observed indicating no significant matrix effect. Fig. S16 (supplementary data) displays the linear regression, the slope, the $y$-intercept and the determination coefficient $\left(R^{2}\right)$ for PG3+1C18:1. The linearity was calculated over three orders of magnitude of concentration resulting in excellent linearity with the $R^{2}$ value of 0.9997 . The RSD were in the range 4.1-13.6\% at 6 different levels of concentration. The limit of detection (LOD) and the limit of quantification (LOQ) were 0.250 and $0.625 \mathrm{pg}$ injected, respectively. For intra-day study performed at six concentrations, the precisions were between $4.1 \%$ and $13.6 \%$, and the accuracies were between $89.2 \%$ and $115.3 \%$. Concerning inter-day data, the precisions were in the range $1.1 \%-6.7 \%$, and the accuracies were between $89.2 \%$ and $115.3 \%$ (Table S2, supplementary data). These results show that our method exhibits good repeatability.

The method was applied to quantify PG3+1C18:1 in three products PG3MO, PG3DO1 and PG3DO2 leading to content of 8.1, 7.4 and $7.4 \%$ of PG3+1C18:1, respectively.

\section{Conclusions}

A rapid and efficient method for characterization of PGEs using SFC-HRMS/MS was established after optimizing SFC and MS parameters. After a single HPLC purification step, pure PG3+1C18:1 was obtained allowing to confirm its structure by HRMS and NMR analyses. For the first time, a method of quantification for PG3+1C18:1 was validated showing a good linearity $\left(R^{2}=0.9997\right)$ as well as sensitivity (pg level). This method was then applied to quantify PG3+1C18:1 in three commercial samples allowing to better characterization of their fine composition. Using the same methodology, other PGE standards could be isolated for a more complete quantification of constituents of commercial PGE mixtures by SFC-HRMS.

\section{Acknowledgments}

We sincerely thank Dr. Véronique Eparvier, Dr. Nathalie Hue and Vincent Steinmetz for allowing us to use the preparative HPLC and SFC systems. 


\section{Formatting of funding sources}

This work was supported by the Agence Nationale de la Recherche (Grant ANR-16-CE29-0002-01 CAPSFC-MS), a grant from Région Ile-de-France (DIM Analytics) and the project funded by the Gattefossé company.

\section{Conflict of Interest}

The authors declare no conflict of interest.

\section{References}

[1] R.T. Mclntyre, Polyglycerol esters, J. Am. Oil Chem. Soc., 56 (1979) 835A-840A. https://doi.org/10.1007/BF02667458.

[2] V.K. Babayan, T.G. Kaufman, H. Lehman, R.J. Tkaczuk, Some uses and applications of polyglycerol esters in cosmetic and pharmaceutical preparations, J. Soc. Cos. Chem., 15 (1964) 473-483.

[3] W. Hemker, Associative structures of polyglycerol esters in food emulsions, J. Am. Oil Chem. Soc., 58 (1981) 114-119. https://doi.org/10.1007/BF02672194.

[4] A.B. Leoneti, V. Aragão-Leoneti, S.V.W. Borges de Oliveira, Glycerol as a by-product of biodiesel production in Brazil: Alternatives for the use of unrefined glycerol, Renew. Energ., 45 (2012) 138-145. https://doi.org/10.1016/j.renene.2012.02.032.

[5] V.K. Babayan, R.T. McIntyre, Preparation and properties of some polyglycerol esters of short and medium chain length fatty acids, J. Am. Oil Chem. Soc., 48 (1971) 307-309. https://doi.org/10.1007/BF02890751.

[6] J.-C. Garrigues, M. Cournac, M. Oswald, N. Ritter, M. Blanzat, S. Cassel, Analysis of complex mixtures of polyglycerol fatty esters using liquid chromatography and high-resolution mass spectrometry: Retention, structural and relative composition study, J. Chromatogr. A, (2020) 1616:460792. https://doi.org/10.1016/j.chroma.2019.460792.

[7] S. Cassel, P. Chaimbault, C. Debaig, T. Benvegnu, S. Claude, D. Plusquellec, P. Rollin, M. Lafosse, Liquid chromatography of polyglycerol fatty esters and fatty ethers on porous graphitic carbon and octadecyl silica by using evaporative light scattering detection and mass spectrometry, J. Chromatogr. A, 919 (2001) 95-106. https://doi.org/10.1016/S0021-9673(01)00801-9.

[8] W.R. Michael, R.H. Coots, Metabolism of polyglycerol and polyglycerol esters, Toxicol. Appl. Pharm., 20 (1971) 334-345. https://doi.org/10.1016/0041-008X(71)90277-8.

[9] H. Nosal, J. Nowicki, M. Warzała, E. Nowakowska-Bogdan, M. Zarebska, Synthesis and characterization of alkyd resins based on Camelina sativa oil and polyglycerol, Prog. Org. Coat., 86 (2015) 59-70. https://doi.org/10.1016/j.porgcoat.2015.04.009.

[10] T.N. Kumar, Y.S.R. Sasatry, G. Lakshminarayana, Analysis of polyglycerols by high performance liquid chromatography, J. Chromatogr. A, (1984) 360-365. https://doi.org/10.1016/S0021-9673(01)927323.

[11] B. De Meulenaer, G.V. Royen, B. Vanhoutte, A. Huyghebaert, Combined liquid and gas chromatographic characterisation of polyglycerol fatty acid esters, J. Chromatogr. A, 896 (2000) 239-251. https://doi.org/10.1016/S0021-9673(00)00391-5.

[12] M. Macka, H.P. Mettler, M. Bokel, W. Röder, Analysis of silanised polyglycerols by supercritical fluid chromatography, J. Chromatogr. A, 675 (1994) 267-270. https://doi.org/10.1016/0021-9673(94)85284-7.

[13] C. Chollet, S. Boutet-Mercey, L. Laboureur, C. Rincon, M. Méjean, J. Jouhet, F. Fenaille, B. Colsch, D. Touboul, Supercritical fluid chromatography coupled to mass spectrometry for lipidomics, J. Mass Spectrom., 54 (2019) 791-801. https://doi.org/10.1002/jms.4445.

[14] E. Lesellier, C. West, The many faces of packed column supercritical fluid chromatography--a critical review, J. Chromatogr. A, 1382 (2015) 2-46. https://doi.org/10.1016/j.chroma.2014.12.083. 
[15] B. Van de Velde, D. Guillarme, I. Kohler, Supercritical fluid chromatography - Mass spectrometry in metabolomics: Past, present, and future perspectives, J. Chromatogr. B Analyt. Technol. Biomed. Life Sci., 1161 (2020) 122444. http://dx.doi.org/10.1016/j.jchromb.2020.122444.

[16] L.C. Harps, J.F. Joseph, M.K. Parr, SFC for chiral separation in bioanalysis, J. Pharm. Biomed. Anal., 162 (2019) 47-59. https://doi.org/10.1016/j.jpba.2018.08.061.

[17] Y. Jiang, J. Fan, R. He, D. Guo, T. Wang, H. Zhang, W. Zhang, High-fast enantioselective determination of prothioconazole in different matrices by supercritical fluid chromatography and vibrational circular dichroism spectroscopic study, Talanta, $187 \quad$ (2018) 40-46. https://doi.org/10.1016/j.talanta.2018.04.097.

[18] D. MacDougall, F.J. Amore, G.V. Cox, D.G. Crosby, F.L. Estes, D.H. Freeman, W.E. Gibbs, G.E. Gordon, L.H. Keith, J. Lal, R.R. Langner, N.I. McClelland, W.F. Phillips, R.B. Pojasek, R.E. Sievers, Guidelines for data acquisition and data quality evaluation in environmental chemistry, Anal. Chem., 52 (1980) 2242-2249. https://doi.org/10.1021/ac50064a004.

[19] T.P.T. Hoang, M. Barthélemy, R. Lami, D. Stien, V. Eparvier, D. Touboul, Annotation and quantification of $\mathrm{N}$-acyl homoserine lactones implied in bacterial quorum sensing by supercritical-fluid chromatography coupled with high-resolution mass spectrometry, Anal. Bioanal. Chem., 412 (2020) 22612276. https://doi.org/10.1007/s00216-019-02265-4.

[20] L. Akbal, G. Hopfgartner, Hyphenation of packed column supercritical fluid chromatography with mass spectrometry: where are we and what are the remaining challenges?, Anal. Bioanal. Chem., (2020). https://doi.org/10.1007/s00216-020-02715-4.

[21] C. Marques-Alvarez, E. Sastre, J. Perez-Pariente, Solid catalysts for the synthesis of fatty esters of glycerol, polyglycerols and sorbitol from renewable resources, Top. Catal., 27 (2004) 105-117. https://doi.org/10.1023/B:TOCA.0000013545.81809.bd. 


\section{Figure captions}

Fig. 1. Chemical structure of PGEs $\left(R^{1}=R^{2}=R^{3}=R^{4}=H\right.$ or acyl group).

Fig. 2. Base peak chromatograms of PG3DO2 solution analyzed by SFC-HRMS with 9 different stationary phases: Hypercarb, PFP (pentafluorophenyl-bonded silica), CN (cyano-bonded silica), C18 (octadecyl carbon-bonded silica), 2-EP (2-ethylpyridine-bonded silica), 1-AA (1-aminoanthracene-bonded silica), DP (diphenyl-bonded silica), $\mathrm{NH}_{2}$ (amino-bonded silica) and DEA (diethylamine-bonded silica). Analysis conditions: The mobile phase was composed of $\mathrm{CO}_{2}(\mathrm{~A})$ and MeOH/EtOH 1:1 v/v with $20 \mathrm{mM}$ of $\mathrm{AcONH}_{4}$ (B) at $1 \mathrm{~mL} \mathrm{m^{-1 }}$ flow rate. The gradient was: 0-10 min (1-30\% B), 10-12 $\min (30 \% \mathrm{~B}), 12-13 \mathrm{~min}(30-$ $1 \% \mathrm{~B}), 13-16 \mathrm{~min}(1 \% \mathrm{~B})$. The temperature of column was $50{ }^{\circ} \mathrm{C}$. The BPR was set at 130 bar and $60{ }^{\circ} \mathrm{C}$. The make-up solvent was $\mathrm{MeOH} / \mathrm{EtOH} \mathrm{1:1} \mathrm{v/v} \mathrm{with} 20 \mathrm{mM}^{\circ} \mathrm{AcONH}_{4}$ at $0.2 \mathrm{~mL} \mathrm{~min}^{-1}$ flow rate.

Fig. 3. EIC of a) monoesters of polyglycerol having $n(n=2-6)$ degree of polymerization and oleic acid (number above each peak corresponds to $n$ value); $b)$ esters of triglycerol $(n=3)$ and $m$ oleic acid $(m=1-5)$ (number above each peak corresponds to $\mathrm{m}$ value); c) PG3+1C18:1 and its analog with one water loss PG3(1)+C18:1. Analysis conditions: The DEA column was set at $50{ }^{\circ} \mathrm{C}$. The mobile phase was composed of $\mathrm{CO}_{2}$ (A) and $\mathrm{MeOH} / \mathrm{EtOH} \mathrm{1:1} \mathrm{v/v} \mathrm{with} 20 \mathrm{mM}$ of $\mathrm{AcONH}_{4}$ (B) at $1 \mathrm{~mL} \mathrm{~min}^{-1}$ flow rate. The gradient was: 0-10 $\min (1-30 \% \mathrm{~B}), 10-12 \min (30 \% \mathrm{~B}), 12-13 \mathrm{~min}(30-1 \% \mathrm{~B}), 13-16 \mathrm{~min}(1 \% \mathrm{~B})$. The BPR was set at $130 \mathrm{bar}$ and $60{ }^{\circ} \mathrm{C}$. The make-up solvent was $\mathrm{MeOH} / \mathrm{EtOH} \mathrm{1:1} \mathrm{v/v} \mathrm{with} 20 \mathrm{mM}$ of $\mathrm{AcONH}_{4}$ at $0.2 \mathrm{~mL}$ $\min ^{-1}$ flow rate.

Fig. 4. Influence of the oven temperature (a) and the BPR (b) on the signal of PG3+1C18:1.

Fig. 5. Influence of the drying-gas temperature (a), the Vcap (b), the fragmentor voltage (c) and the nozzle voltage (d) on the signal of PG3+1C18:1.

Fig.6. HRMS/MS spectra (10 eV of collision energy) of the most intense PG3+1C18:1 isomer with MS/MS fragmentation pattern suggested for a linear PG3+1C18:1 with the fatty chain positioned in terminal position of PG3 $(\mathrm{G}=$ glycerol, FA = fatty acid, PG(2)1 = diglycerol with one water loss, PG3(1) = triglycerol with one water loss). 


\section{Table}

Table 1. ${ }^{1} \mathrm{H}$ and ${ }^{13} \mathrm{C}$ NMR data for PG3+1C18:1.

\begin{tabular}{|c|c|c|c|}
\hline \multirow{2}{*}{ Position } & \multicolumn{3}{|c|}{ PG3+1C18:1 } \\
\hline & Type & $\delta \mathrm{C}(\mathrm{ppm})$ & $\delta_{\mathrm{H}}(\mathrm{ppm}, \mathrm{J}$ en Hz$)$ \\
\hline 1 & $\mathrm{C}$ & 175.6 & \\
\hline 2 & $\mathrm{CH}_{2}$ & 35.1 & $2.33-2.37(\mathrm{~m})$ \\
\hline 3 & $\mathrm{CH}_{2}$ & 26.2 & $1.57-1.66(\mathrm{~m})$ \\
\hline 4 & $\mathrm{CH}_{2}$ & 30.4 & $1.25-1.39(\mathrm{~m})$ \\
\hline 5 & $\mathrm{CH}_{2}$ & 30.3 & $1.25-1.39(\mathrm{~m})$ \\
\hline 6 & $\mathrm{CH}_{2}$ & 30.5 & $1.25-1.39(\mathrm{~m})$ \\
\hline 7 & $\mathrm{CH}_{2}$ & 31.0 & $1.25-1.39(\mathrm{~m})$ \\
\hline 8 & $\mathrm{CH}_{2}$ & 28.3 & $2.00-2.07(\mathrm{~m})$ \\
\hline 9 & $\mathrm{CH}$ & 131.1 & $5.32-5.38(\mathrm{~m})$ \\
\hline 10 & $\mathrm{CH}$ & 131.0 & $5.32-5.38(\mathrm{~m})$ \\
\hline 11 & $\mathrm{CH}_{2}$ & 28.3 & $2.00-2.07(\mathrm{~m})$ \\
\hline 12 & $\mathrm{CH}_{2}$ & 30.9 & $1.25-1.39(\mathrm{~m})$ \\
\hline 13 & $\mathrm{CH}_{2}$ & 30.8 & $1.25-1.39(\mathrm{~m})$ \\
\hline 14 & $\mathrm{CH}_{2}$ & 30.6 & $1.25-1.39(\mathrm{~m})$ \\
\hline 15 & $\mathrm{CH}_{2}$ & 30.3 & $1.25-1.39(\mathrm{~m})$ \\
\hline 16 & $\mathrm{CH}_{2}$ & 33.2 & $1.25-1.39(\mathrm{~m})$ \\
\hline 17 & $\mathrm{CH}_{2}$ & 23.9 & $1.25-1.39(\mathrm{~m})$ \\
\hline 18 & $\mathrm{CH}_{3}$ & 14.6 & $0.90(\mathrm{t}, 6.9)$ \\
\hline $1^{\prime}$ & $\mathrm{CH}_{2}$ & 66.7 & 4.06-4.17 (m) \\
\hline $2^{\prime}$ & $\mathrm{CH}$ & 69.8 & $3.92-3.97(\mathrm{~m})$ \\
\hline $3^{\prime}$ & $\mathrm{CH}_{2}$ & 73.8 & $3.49-3.58(\mathrm{~m})$ \\
\hline $4^{\prime}$ & $\mathrm{CH}_{2}$ & 74.1 & $3.49-3.58(\mathrm{~m})$ \\
\hline $5^{\prime}$ & $\mathrm{CH}$ & 70.9 & $3.87-3.92(\mathrm{~m})$ \\
\hline $6^{\prime}$ & $\mathrm{CH}_{2}$ & 74.0 & $3.49-3.58(\mathrm{~m})$ \\
\hline $7^{\prime}$ & $\mathrm{CH}_{2}$ & 72.8 & $3.56-3.64(\mathrm{~m})$ \\
\hline $8^{\prime}$ & $\mathrm{CH}$ & 72.4 & $3.73-3.79(\mathrm{~m})$ \\
\hline $9^{\prime}$ & $\mathrm{CH}_{2}$ & 64.5 & $3.50-3.59(\mathrm{~m})$ \\
\hline
\end{tabular}

